\title{
Ferromagnetic transition in dilute Pd-Fe alloys
}

\author{
C. Büscher, T. Auerswald, E. Scheer, A. Schröder, and H. v. Löhneysen \\ Physikalisches Institut, Universität Karlsruhe, 7500 Karlsruhe, Germany
}

\author{
H. Claus* \\ Kernforschungszentrum Karlsruhe, Institut für Technische Physik, Postfach 3640, 7500 Karlsruhe, Germany \\ and Physikalisches Institut, Universität Karlsrhue, 7500 Karlsruhe, Germany \\ (Received 21 August 1991; revised manuscript received 2 March 1992)
}

\begin{abstract}
We present low-field magnetization and specific-heat data for $\mathbf{P d}_{1-x} \mathrm{Fe}_{x}$ alloys, with $x$ ranging from 0.08 to 0.6 at. \% Fe. Both types of measurements were done on the same samples. The samples are more homogeneous than those used in previous investigations. We find that in contrast to previous suggestions, all samples undergo sharp ferromagnetic transitions with $T_{C}$ ranging from $0.4 \mathrm{~K}$ for the 0.08 -at. \% Fe alloy to $18.0 \mathrm{~K}$ for the 0.6 -at. \% Fe alloy. The specific-heat anomalies associated with the transitions have the form of a cusp very close to $T_{C}$ as determined from the magnetization. However, because of large fluctuations, these anomalies are very broad, extending to a region of temperatures well above $T_{C}$. Combining our results with earlier data, we argue that the critical concentration for ferromagnetism in Pd-Fe alloys is near 0.01 at. $\% \mathrm{Fe}$, lower by a factor of 10 than previously assumed.
\end{abstract}

\section{INTRODUCTION}

Dilute Pd-Fe alloys have been studied thoroughly over the last 30 years. It takes only about 0.1 at. $\% \mathrm{Fe}$ for long-range ferromagnetic order to develop. ${ }^{1}$ The individual magnetic moments, which undergo an order-disorder transition at the ferromagnetic Curie temperature $T_{C}$ are giant moments, so-called polarization clouds. The magnetic moments on the iron sites polarize the surrounding $\mathrm{Pd}$ matrix, producing magnetic moments of about $10 \mu_{B}{ }^{1}$ The spin quantum number of these giant moments is only about $S=2$ as estimated from the specific-heat anomaly associated with the ferromagnetic transition. ${ }^{1}$ All these results are discussed in a review article by Nieuwenhuys. ${ }^{1}$ As pointed out there, these early measurements were plagued by rather broad magnetic transitions making it difficult to determine the ferromagnetic Curie temperature. ${ }^{1}$ It was even speculated that these alloys may not be conventional ferromagnets at all, i.e., do not possess a well-defined ordering temperature. ${ }^{1}$ However, we have since demonstrated that carefully prepared $\mathrm{Pd}-\mathrm{Fe}$ alloys do indeed display very sharp ferromagnetic transitions. ${ }^{2}$ The broad transitions seen earlier are probably due to sample inhomogeneities as will be discussed below. The specific-heat anomalies associated with the ferromagnetic transitions, again, were unusually broad, extending over a very large temperature range up to $3 T_{C}$ and more. ${ }^{1}$ Thus, it is desirable to remeasure these anomalies for our more homogeneous samples. In this paper we report specific-heat measurements on homogeneous $\mathrm{Pd}_{1-x} \mathrm{Fe}_{x}$ samples in the range of $0.08 \leq x \leq 0.6$ at. \% Fe. For the same samples we also present low-field dc magnetization data. We are thus able to demonstrate that the cusp in the specific heat of Pd-Fe alloys indeed coincides closely with the magnetically determined Curie temperature, in contrast to some previous assumptions. ${ }^{3}$ It is also found that the specific-heat anomalies associated with the fer- romagnetic transitions are considerably narrower than reported earlier. However, they remain broad, especially at the lowest $\mathrm{Fe}$ concentrations. This effect is attributed to an increasing degree of magnetic short-range order or fluctuations as the Fe concentration is lowered. It is also suggested that the critical concentration for ferromagnetism is about 0.01 at. \% Fe, i.e., an order of magnitude lower than previously assumed.

\section{EXPERIMENTAL}

The specific heat was determined by a standard heatpulse method in a ${ }^{4} \mathrm{He}$ cryostat with the samples being mounted on a sapphire sample holder. ${ }^{4}$ Because of the small size of the samples (see below), the absolute accuracy of the specific-heat measurements is probably not better than $5 \%$. The 0.12 -at. $\% \mathrm{Fe}$ and 0.08 -at. \% Fe samples were measured in a dilution refrigerator with a $\mathrm{Si}$ wafer as sample holder. ${ }^{4}$ The magnetization was measured in fields of typical 1.0 or $0.1 \mathrm{G}$ employing a commercial superconducting quantum interference device (SQUID) probe. ${ }^{5}$ The Earth's magnetic field was shielded by $\mu$ metal to less than $0.01 \mathrm{G}^{5}$

A crucial part of this investigation is the preparation of homogeneous samples, minimizing Fe-concentration gradients as much as possible. Over the years we have developed a technique that quite reproducibly resulted in very homogeneous samples. ${ }^{6}$ The alloys were prepared in an arc furnace in $\mathrm{Ar}$ atmosphere on a water cooled $\mathrm{Cu}$ crucible. A 16-at. \% Fe master alloy was diluted with Pd first to a 3-at. \% Fe alloy. Pieces of this latter alloy were then further diluted with the appropriate amounts of Pd to yield about $2 \mathrm{~g}$ samples with 0.08 to 0.6 at. $\% \mathrm{Fe}$. All samples were remelted at least four times, each time being turned. The samples were then sealed in a quartz capsule under 280-mbar Ar gas together with some Ta foil for oxygen gettering, placed in a furnace at $1230^{\circ} \mathrm{C}$ 
for 5 days. The capsules were then water quenched. The samples were then severely cold worked by compressing them repeatedly in three mutually perpendicular directions each time reducing the linear dimension by $10-20 \%$. They were then resealed in quartz capsules and the whole annealing-quenching-coldworking procedural sequence was repeated three times. Before the last anneal the sample was compressed from initially 5 $\mathrm{mm}$ to a thickness of about $2 \mathrm{~mm}$. After the last $1230^{\circ} \mathrm{C}$ anneal a $2-\mathrm{mm}$ strip was cut from the center of the flat sample and compressed perpendicular to the cutting surfaces to $0.7 \mathrm{~mm}$. From the center of this plate the final sample was cut, a rectangularly shaped platelet of typical $3 \times 8 \times 0.7 \mathrm{~mm}^{3}$ weighing about $200 \mathrm{mg}$. This cutting procedure ensured that the final sample does not contain any material that was close to the surface during the high-temperature anneals, thus, avoiding concentration gradients arising from preferential evaporation at high temperatures. The corners of the samples were then rounded by grinding to mimic a roughly ellipsoidal shape. The samples were then chemically etched and given a final $30-\mathrm{min}$ anneal at $950^{\circ} \mathrm{C}$ to relieve all strain. ${ }^{6,7}$

Figure 1 displays low-field magnetization data for a 0.5 -at. \% Fe sample prepared in the way described above, but weighing only about $25 \mathrm{mg}$. The response shown is that of an ideal soft ferromagnet. ${ }^{6,8}$ At a temperature $T^{*}$, sometimes referred to as kink temperature, which depends on the field, the magnetic susceptibility reaches the demagnetization limit of $1 / N(N=$ demagnetization factor) and remains at this limit down to the lowest temperatures. ${ }^{6,8}$ The slight increase in the susceptibility below the kink temperature $T^{*}$ (Fig. 1) is due to a small hysteresis that keeps the magnetization slightly above its thermodynamic equilibrium value of $M=H / N$ $(H=$ applied field $){ }^{8}$ Extrapolating the kink temperature $T^{*}(H)$ to zero field yields the Curie temperature of $T_{C}=14.2 \mathrm{~K}$. Actually, $0.1 \mathrm{G}$ is small enough so that $T^{*}(0.1 \mathrm{G})=T_{C}$ within a few hundreds of a degree. At 10

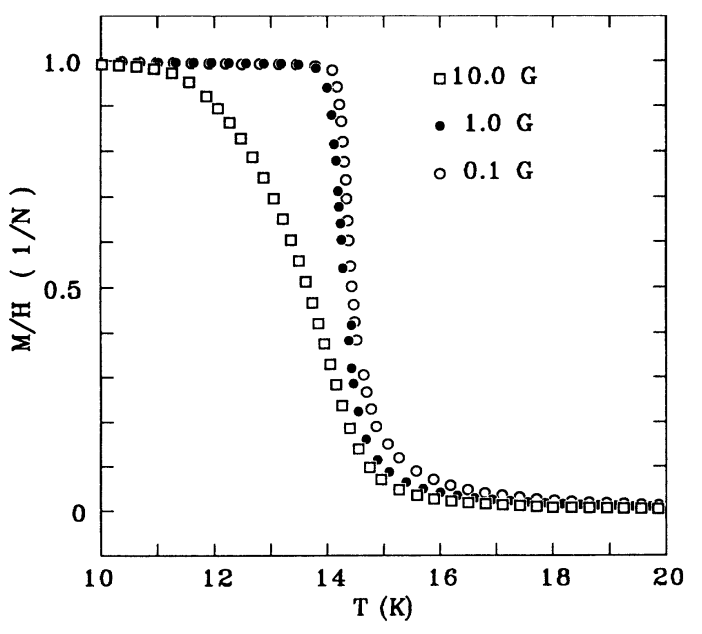

FIG. 1. Magnetic susceptibility $M / H$ in units of the reciprocal demagnetization factor $1 / N$ vs temperature $T$ for a 0.5 -at. \% Fe sample measured in a magnetic field of $0.1,1.0$, and $10 \mathrm{G}$.
$\mathrm{G}$, however, the transition is already considerably rounded and thus very low fields are required to determine $T_{C}$ correctly. The demagnetization factor determined from the geometry of the samples agreed with that determined from the magnetization measurements to within $10 \%$. The difference can be explained in terms of absolute accuracy of the SQUID magnetometer and also sample shape irregularities (deviation from the ideal ellipsoidal form). For the magnetization units in Figs. 1 and 2, we chose $N$ as determined from magnetization measurements, i.e., those units are to some degree arbitrary.

In $0.1 \mathrm{G}$ the width of the transition (Fig. 1) is extremely narrow. We estimate that the broadening due to $\mathrm{Fe}$ concentration gradients (if any) has to be less than a few tenths of a degree. With a concentration dependence of $T_{C}$ about $40 \mathrm{~K} /$ at. $\% \mathrm{Fe}$ (Ref. 8 ) this yields a homogeneity of better than $1 \%$, i.e., better than 0.005 at. $\%$ Fe. This extreme homogeneity was obtainable only for small samples of less than about $30 \mathrm{mg}$. For the specific-heat measurements, however, much larger samples of about $200 \mathrm{mg}$ are required and thus somewhat larger inhomogeneities had to be tolerated.

\section{RESULTS AND DISCUSSION}

\section{A. Magnetization}

Typical magnetization measurements on the samples used for the specific heat measurement are shown in Fig. 2. Shown is the magnetization in a field of $0.1 \mathrm{G}$ measured with increasing temperature after initially cooling in $0.1 \mathrm{G}$ (open circles) and in zero field (closed circles). The transitions are somewhat broader than that of the smaller more homogeneous sample shown in Fig. 1. We define $T_{C}$ from the intercept of the extrapolations of the linear behavior of $M$ below and above the transition as demonstrated for the 0.36-at. \% Fe sample in Fig. 2. For the lower $\mathrm{Fe}$ concentrations, hysteresis develops causing a separation of the zero-field-cooled and field-cooled magnetization. As mentioned above, this hysteresis is due to a small pinning of domain walls keeping the magnetization either above or below the thermodynamic equilibrium value of $H / N \quad(H=$ applied field and $N=$ demagnetization factor) ${ }^{8}$ For some of the samples in Fig. 2, especially for that with 0.36 at. \% Fe, the zerofield-cooled and field-cooled branch merge above the $\mathrm{Cu}$ rie temperature. This is a clear sign of an inhomogeneous sample, with parts of the sample still being ferromagnetic (with hysteresis) above the Curie temperature of the bulk. For a homogeneous sample the two branches would meet right at $T_{C}$, both branches reaching a magnitude of $H / N$ as we have shown before. ${ }^{8}$ The inhomogeneities of the samples of Fig. 2 cause a smearing of their ferromagnetic transitions of typically much less than one degree, still considerably smaller than earlier measurements. ${ }^{1}$ The 0.5 -at. \% Fe sample clearly is inhomogeneous, displaying a steplike structure in $M$ vs $T$. This sample consisted of two separate pieces of the same nominal composition prepared in the same run, which when measured separately turned out to have two distinct Curie temperatures, one with $T_{C}=12.8 \mathrm{~K}$, the other with $14.0 \mathrm{~K}$. Fig- 
ure 3 shows magnetization data for the 0.08-at. \% Fe alloy. These data were taken in a dilution-refrigerator vibrating-sample magnetometer. ${ }^{9}$ Only relative values of $M$ could be determined. Thus we are not able to determine from magnetization alone if this sample is indeed ferromagnetic (but see Sec. III B below).

Figure 4 displays the Curie temperatures determined in the present investigation and also some earlier results ${ }^{10}$ for larger Fe concentrations. Also shown are the temperatures of the maxima in the specific-heat anomalies (see below). The present data and some results from earlier investigations extending over a larger $x_{\mathrm{Fe}}$ range are listed in Table I. As has been observed for many other transition metal alloys, ${ }^{6,8} T_{C}$ varies roughly linear with concentration in the range 0.3 at. $\% \leq x_{\mathrm{Fe}} \leq 1.0$ at. $\%$ (Fig. 4). The extrapolation of this linear part to the concentration axis yields a concentration that is often referred to as
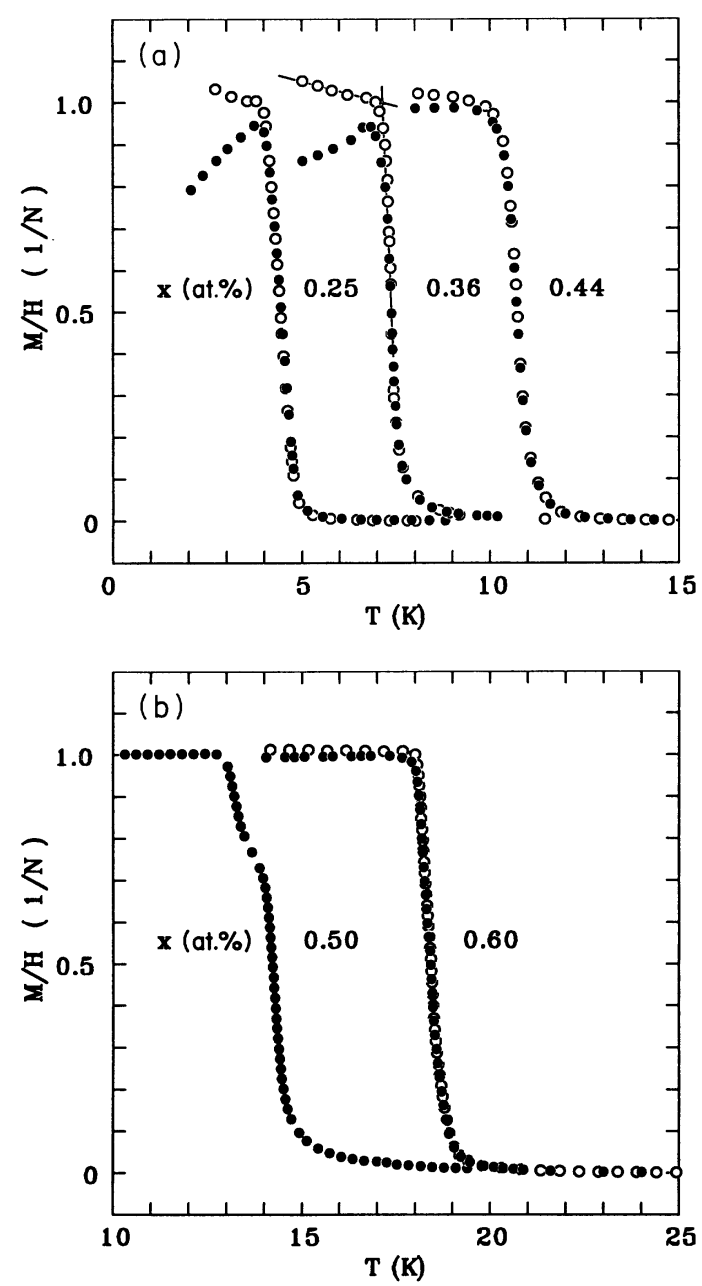

FIG. 2. Magnetic susceptibility $M / H$ in units of the reciprocal demagnetization factor $1 / N$ vs temperature $T$ for various Pd-Fe samples used for the specific-heat measurements. The numbers labeling the curves are the Fe concentrations in at. $\%$. The measuring field is $H=0.1 \mathrm{G}$. For the 0.36 -at. \% Fe alloy it is demonstrated how $T_{C}$ is determined (from the intersection of the lines drawn through the data points).

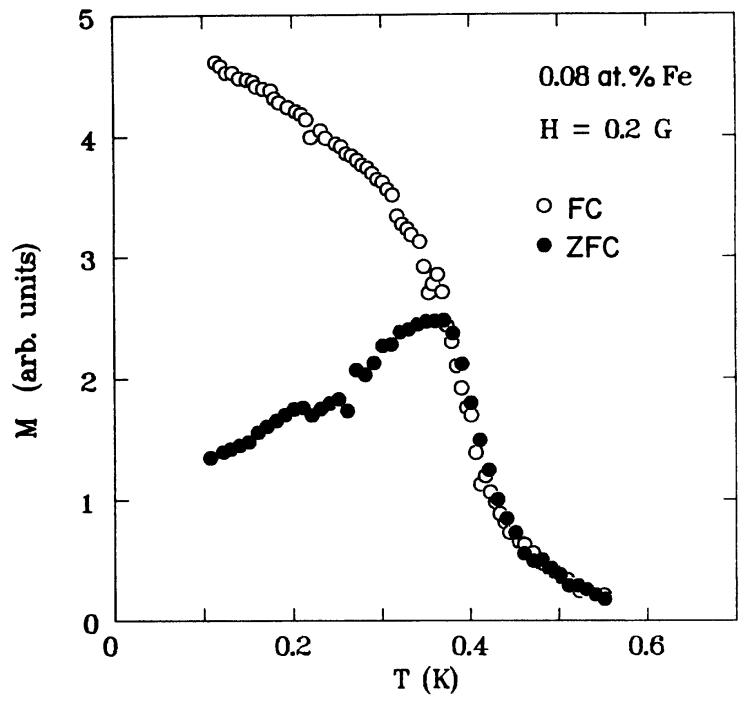

FIG. 3. Magnetization in arbitrary units vs temperature in a field of $0.2 \mathrm{G}$ for the sample with 0.08 at. \% Fe.

critical concentration $x_{C}=0.19$ at. $\%$ Fe. The exact value of this parameter depends to some degree on the range of $x_{\mathrm{Fe}}$ used to define the linear behavior. There are strong deviations seen from the linear behavior both at low concentration (Fig. 4) and also higher concentrations ${ }^{14}$ (now shown). A much better fit to the $T_{C}$ vs $x_{\mathrm{Fe}}$ data can be achieved, using a relation based on the percolation approach, ${ }^{15} T_{C}=A \exp \left(-B x_{\mathrm{Fe}}^{-1 / 3}\right)$. This expression yields an excellent fit to the data in the concentration range $0.08 \leq x_{\mathrm{Fe}} \leq 3.2$ at. $\% \mathrm{Fe}$, but $T_{C}$ values for $x_{\mathrm{Fe}}<0.08$ at. \% Fe (Refs. 10-12) lie considerably above this expression (dashed line in Fig. 5).

It is at present not clear how far down in $x_{\mathrm{Fe}}$ fer-

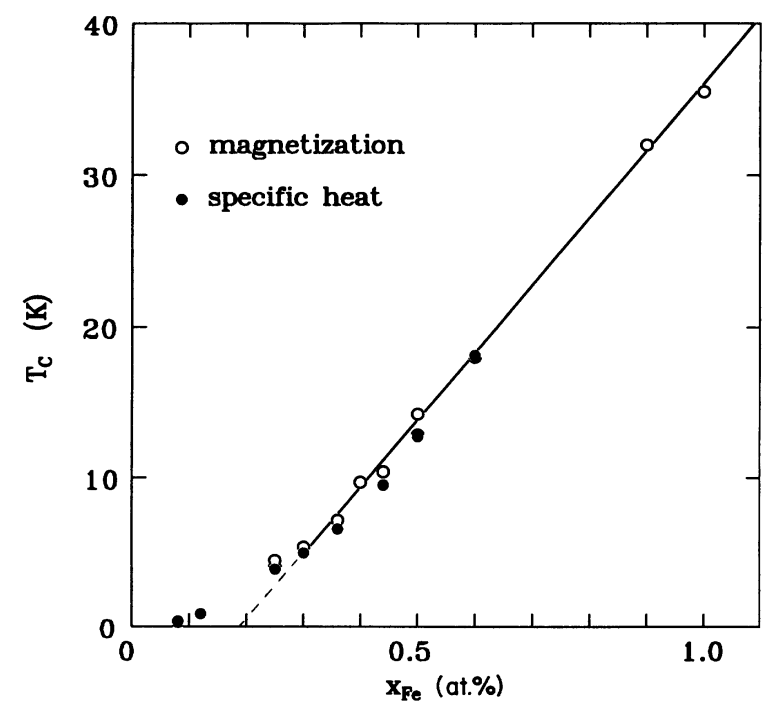

FIG. 4. Magnetic ordering temperature $T_{C}$ determined from magnetization (open circles) and specific heat (full circles) vs $\mathrm{Fe}$ concentration (see Table I). 
romagnetism actually exists in Pd-Fe. At very low concentrations, $x_{\mathrm{Fe}}<300 \mathrm{ppm}$, spin-glass behavior has been reported. ${ }^{10,11}$ The criterion used there to demonstrate spin-glass behavior was the observation of a cusp in the ac susceptibility. However, any ferromagnet with hysteresis developing right at $T_{C}$ displays a cusp in the ac susceptibility similar in shape to the zero-field-cooled branch of the dc magnetization. $^{2}$ The 0.25 -at. \% Fe sample in Fig. 2, for example, would display a sharp cusp in the ac susceptibility but is definitely ferromagnetic. Therefore, a cusp in the ac susceptibility is not sufficient to prove spin-glass behavior.

It has been observed previously in other transition metal alloys like $\mathrm{Cu}-\mathrm{Ni}, \mathrm{Rh}-\mathrm{Ni}, \mathrm{Au}-\mathrm{Fe}$, and $\mathrm{V}-\mathrm{Fe}$, that the change over from ferromagnetic to spin-glass ordering as the concentration is reduced is associated with a sudden decrease of $\chi_{M}$, the height of the ac susceptibility cusp. $^{6,16}$ In the ferromagnetic regime, $\chi_{M}=1 / N$, independent of $T_{C}$ (i.e., concentration). As spin-glass ordering sets in, this height decreases fast as the concentration is further reduced. Indeed, this decrease in height has been observed in Pd-Fe for $x_{\mathrm{Fe}} \leq 100 \mathrm{ppm}$. $^{10}$ However, as was shown previously, a drastic decrease in the height of the cusp can also occur in a ferromagnet due to increasing inhomogeneity. ${ }^{8}$ For example, it was demonstrated $^{8}$ that, for a slightly inhomogeneous ferromagnetic Pd-0.5-at. \% Co sample, the height of the cusp was only about $20 \%$ of $1 / N$ (Ref. 8). Larger inhomogeneities could decrease this height even further. ${ }^{8}$ The only decisive test for ferromagnetism in case of hysteresis from macroscopic measurements are field-cooled dc magnetization measurements in sufficiently small fields. In case

TABLE I. Magnetic ordering temperatures $T_{C}$, determined from both magnetization $M(T)$, and specific heat $C(T)$, for various dilute $\mathrm{Pd}_{1-x} \mathrm{Fe}_{x}$ samples.

\begin{tabular}{lccl}
\hline \hline & $\begin{array}{c}T_{C}(\mathrm{~K}) \\
x_{\mathrm{Fe}} \text { (at. \%) }\end{array}$ & $\begin{array}{c}T_{C}(\mathrm{~K}) \\
\text { from } M(T)\end{array}$ & \multicolumn{1}{c}{ Source } \\
\hline 0.00018 & 0.00018 & & Ref. 11 \\
0.00078 & 0.00049 & & Ref. 11 \\
0.0014 & 0.0009 & & Ref. 11 \\
0.010 & 0.009 & & Ref. 11 \\
0.0145 & 0.018 & & Ref. 12 \\
0.022 & 0.041 & & Ref. 12 \\
0.070 & 0.32 & & Ref. 13 \\
0.080 & 0.36 & 0.37 & Present work \\
0.12 & & 0.88 & Present work \\
0.25 & 4.1 & 3.90 & Present work \\
0.25 & 4.5 & & Ref. 10 \\
0.30 & 5.4 & 5.0 & Present work \\
0.36 & 7.2 & 6.6 & Present work \\
0.40 & 9.7 & & Ref. 2 \\
0.44 & 10.4 & 9.5 & Present work \\
0.50 & 12.8 & 12.7 & Present work \\
0.50 & 14.2 & & Ref. 10 \\
0.60 & 18.0 & 18.1 & Present work \\
0.90 & 32.0 & & Ref. 10 \\
1.00 & 35.5 & & Ref. 10 \\
3.20 & 101.5 & & Ref. 10 \\
\hline \hline
\end{tabular}

of ferromagnetic order (in the presence of hysteresis) the field-cooled branch of $M$ vs $T$ will rise well above the demagnetization limit of $H / N{ }^{2,8}$

Another effect, associated with the change from ferromagnetic to spin-glass order as the concentration is reduced, is a sudden drastic change in the concentration dependence of the ordering temperature. This is seen in many transition-metal alloys. ${ }^{16}$ As an example, the rate at which the ordering temperature changes with concentration in both $\mathrm{Cu}-\mathrm{Ni}$ and $\mathrm{V}$-Fe alloys, decreases by about a factor of 10 as the nature of the ordering changes from ferromagnetic to spin glass. ${ }^{6,16}$ The detection of such a behavior in $\mathrm{Pd}-\mathrm{Fe}$ alloys from the earlier data was not possible due to the large scatter. In a more recent investigation, however, it has been reported that for $x_{\mathrm{Fe}} \leq 100$ ppm the ordering temperature varies approximately linearly with $x_{\mathrm{Fe}} \cdot{ }^{10}$ Thus, it will be instructive to plot $T_{C}$ vs $x_{\mathrm{Fe}}$ on a double logarithmic plot as is done in Fig. 5. In addition to our own data, Fig. 5 also displays data for very low concentrations from other groups, all done fairly recently in a dilution refrigerator (see Table I). Earlier data from Ref. 1 are not included in Fig. 5 because of their large scatter. Within the uncertainty in the concentration, $T_{C}$ varies linearly with $x_{\mathrm{Fe}}$ up to about $100 \mathrm{ppm}$ Fe. ${ }^{11}$ As the concentration increases further, there is a sudden change to an approximate $x_{\mathrm{Fe}}^{2}$ dependence up to about 1 at. $\% \mathrm{Fe}$, above which $T_{C}$ again varies linearly in $x_{\mathrm{Fe}}$. The nearly quadratic behavior at intermediate concentrations and the change to a linear dependence above 1 at. \% Fe can be quantitatively explained in a simple model, where $T_{C}$ is proportional to the amount of direct overlap of the polarization clouds. ${ }^{1,17}$ The behavior as seen in Fig. 5 is consistent with the early $T_{C}$ data of Ref. 1 , except that those early data for very low Fe concentrations and also more recent results on a $3.2 \mathrm{ppm} F e$ sample $^{18}$ are considerably higher than the data of Fig. 5, a

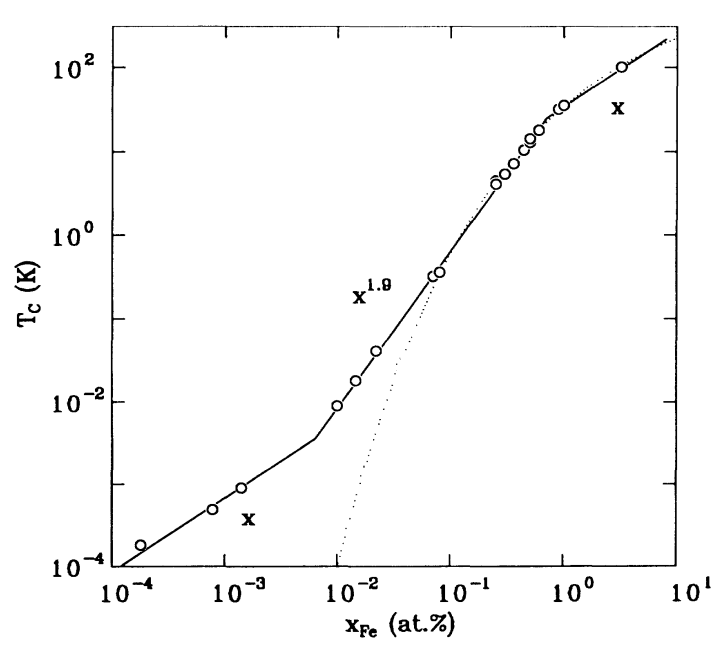

FIG. 5. Magnetic ordering temperature $T_{C}$ as determined from magnetization measurements of various authors (Table I) on a double-logarithmic scale. The solid lines through the data points have slopes of $1,1.9$, and 1 as indicated. The dashed line is a fit to $T_{C}=A \exp \left(-B x_{\mathrm{Fe}}^{-1 / 3}\right)$ (see text). 
fact which we do not understand. Without real justification we will exclude these earlier results from our discussion.

If the amount of the direct overlap of the polarization clouds is responsible for the ferromagnetic order, ${ }^{17}$ then the nature of the ordering should change when the average separation of the $\mathrm{Fe}$ atoms becomes larger than the diameter of the clouds. We suggest that this happens near $x_{\mathrm{Fe}}=0.01$ at. $\% \mathrm{Fe}$, the concentration where $T_{C}$ vs $x_{\mathrm{Fe}}$ changes from linear to near quadratic behavior (Fig. 5). Indeed, at this concentration the average distance between $\mathrm{Fe}$ atoms is about $5 \mathrm{~nm}$, which is practically identical with the diameter of the polarization clouds determined from diffuse neutron scattering. ${ }^{19}$ Only for concentrations less than $100 \mathrm{ppm}$ there is firm experimental evidence suggesting spin-glass ordering ${ }^{11}$ (from the reduction of the height of the cusp). No such evidence is available for an alloy with $220 \mathrm{ppm}$, which was judged to be a spin glass solely by the existence of the ac susceptibility cusp. ${ }^{12}$ As pointed out above, this is a necessary but not sufficient criterion for spin-glass behavior. An alloy with 700 ppm Fe was judged to be ferromagnetic from dc magnetization measurements. ${ }^{13}$ The critical concentration for ferromagnetism suggested above, $x_{C}=100 \mathrm{ppm} \mathrm{Fe}$ is about one order of magnitude smaller than previously assumed. 1,20 However, more dc magnetization measurements for $x_{\mathrm{Fe}}<0.1$ at. \% will be required to check this assumption.

\section{B. Specific heat}

Typical specific heat results are displayed in Fig. 6 for pure $\mathrm{Pd}$ and two $\mathrm{Pd}_{1-x} \mathrm{Fe}_{x}$ alloys for $x=0.25$ at. $\% \mathrm{Fe}$ and $x=0.44$ at. $\%$ Fe. Specific-heat measurements were performed in zero magnetic field and also in a field of $H=6 \mathrm{~T}$. As can be seen in Fig. 6, the magnetic field has no effect on the specific heat of Pd in agreement with earlier measurements. ${ }^{21}$ The low temperature part of the $\mathrm{Pd}$ data $(T \leq 10 \mathrm{~K})$ can be well represented by $C / T=\gamma+\beta T^{2}$, yielding $\gamma=9.47 \mathrm{~mJ} / \mathrm{mol} \mathrm{K}^{2}$ and $\beta=0.093 \mathrm{~mJ} / \mathrm{mol} \mathrm{K}^{4}$. Within our accuracy, these values are in good agreement with earlier results. ${ }^{1,3,21}$ For the two alloys shown in Fig. 6, the magnetic anomalies associated with the ferromagnetic transition $\left(T_{C}\right.$ as determined from magnetic data are marked by the arrows) are clearly visible in the zero-field measurements. However, this anomaly is less visible for the higher $\mathrm{Fe}$ concentrations due to the increasing magnitude of the electronic $(\gamma T)$ and lattice $\left(\beta T^{3}\right)$ specific heat. In $H=6 \mathrm{~T}$, the anomaly near $T_{C}$ is wiped out. As expected, the entropy change associated with the disordering of the magnetic moments is now shifted to higher temperatures, as can be seen from the fact that at these higher temperatures the specific heat in $H=6 \mathrm{~T}$ is larger than in zero field (Fig. 6). However, a magnetic field of $6 \mathrm{~T}$ is obviously much too small to shift all magnetic degrees of freedom above our range of temperatures. This would have been desirable for subtracting the electronic and lattice specific heat, which, as we know for pure Pd, is not affected by the magnetic field. ${ }^{21}$ Thus, in order to obtain the specific-heat anomaly associated with the ferromagnetic transition, we assume, as was done previously, ${ }^{1,3}$ that the lattice and electronic terms in the specific heat of dilute $\mathrm{Pd}-\mathrm{Fe}$ alloys are the same as those of pure $\mathrm{Pd}$. Figure 7 shows $\Delta C=C_{\text {alloy }}-C_{\mathrm{Pd}}$ data for the five lowest $\mathrm{Fe}$ concentrations investigated. At temperatures well above the
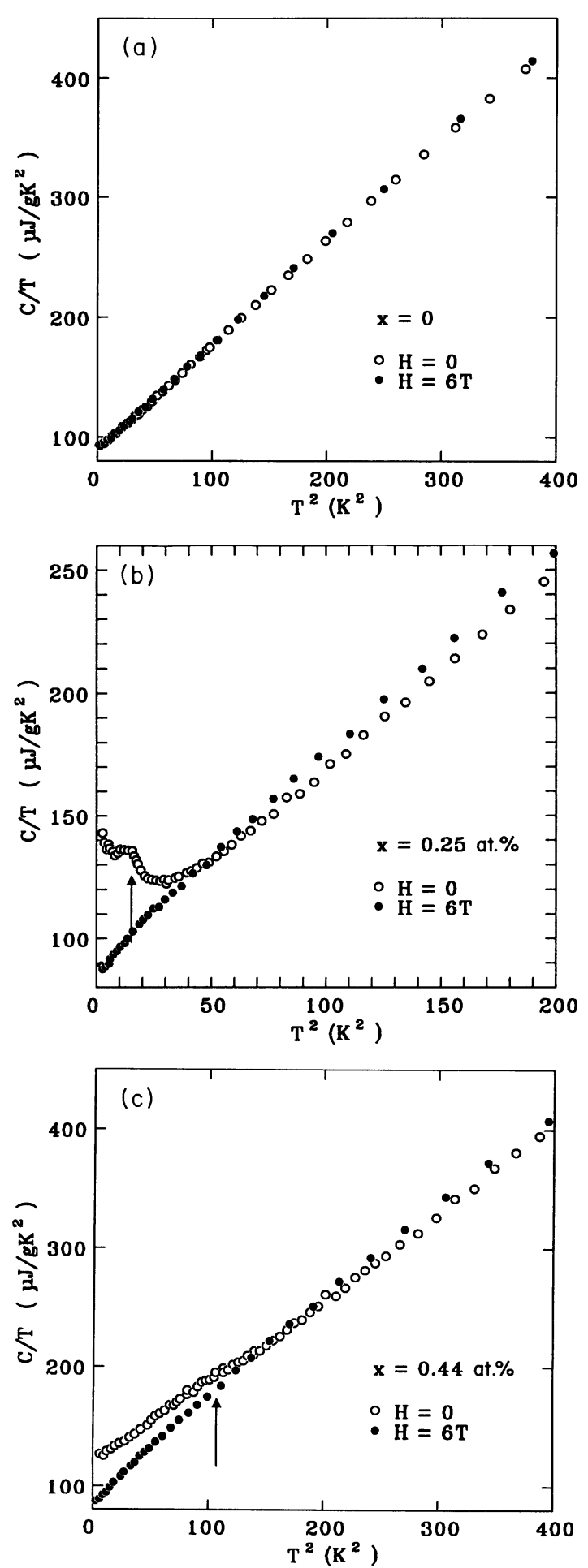

FIG. 6. Specific-heat data in form of $C / T$ vs $T^{2}$ plots for Pd, $\mathrm{Pd}-0.25$ at. $\% \mathrm{Fe}$, and $\mathrm{Pd}-0.44$ at. $\% \mathrm{Fe}$, in zero magnetic field (open circles) and in $H=6 \mathrm{~T}$ (full circles). Arrows indicate the magnetically determined ordering temperatures. 


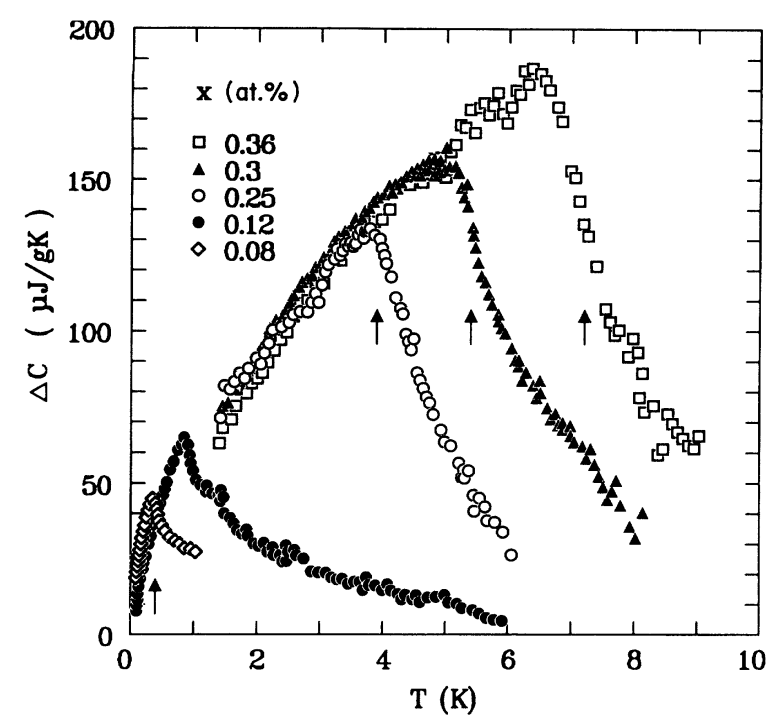

FIG. 7. Excess specific heat $\Delta C=C_{\text {alloy }}-C_{P_{d}}$ vs temperature for the samples with the lowest Fe concentrations. Arrows indicate the magnetically determined ordering temperatures.

cusp, $\Delta C$ increasingly becomes negative (not shown). This is especially true for the higher Fe concentrations. This effect may be due to a decrease in the electronic and lattice specific heat of Pd upon alloying. Also, the magnetic contribution to the total specific heat is rather small, especially for the higher Fe concentrations. Thus, any quantitative determination of the total entropy change due to the magnetic ordering is not possible.

The arrows in Fig. 7 indicate the magnetically determined Curie temperatures (the $0.12 \%$ Fe sample was not measured magnetically). There is close agreement between the Curie temperature and the temperature where the cusp occurs. Despite the very sharp ferromagnetic transitions measured magnetically (Fig. 2), the specificheat anomalies associated with those transitions extend to fairly high temperatures similar to the earlier results. ${ }^{1}$ The relative width of the transitions considerably increases with decreasing concentrations. For 0.36 at. \% Fe we estimate that the excess specific heat due to the ordering process extends to a temperature of about $2 T_{C}$, whereas for the 0.12 -at. \% Fe sample it extends to about $6 T_{C}$ (Fig. 7). This large range of magnetic fluctuations or short-range order is probably due to the statistical nature of the alloys leading to a wide distribution of local atomic environments. However, in contrast to earlier as- sumptions, ${ }^{1}$ it is quite clear from our magnetization measurements that the alloys undergo a well-defined magnetic phase transition.

As mentioned above, Table I also lists the ordering temperatures determined from the specific-heat anomaly. This temperature was taken to be that at which $\Delta C(T)$ first starts decreasing again with increasing temperature (see Fig. 7). This temperature agrees reasonably well with that determined from the magnetization data of Fig. 2, being, however, slightly lower for most alloys (see Fig. 4 and Table I). For the 0.08-at. \% Fe alloy, the rather sharp cusp in $\Delta C(T)$ and the close agreement between the magnetic ordering temperature with the cusp temperature clearly shows that this alloy is still ferromagnetic. It is well known that for spin-glass ordering the specificheat anomaly would occur well above the ordering temperature and would be much more rounded than the anomalies observed here. An interesting observation is the fact that the specific heat below $T_{C}$ follows an almost "universal" curve, indicating that the low-energy spectrum of the magnetic excitations is practically independent of $\mathrm{Fe}$ concentration. Increasing $x_{\mathrm{Fe}}$ merely results in extending the spectrum to higher energies. The origin of this behavior remains to be established.

\section{CONCLUSIONS}

We have presented both specific-heat and low-field magnetization data of Pd-Fe alloys, which were much more homogeneous than in previous investigations. All investigated samples $\left(0.08\right.$ at. $\% \leq x_{\mathrm{Fe}} \leq 0.6$ at. $\left.\%\right)$ undergo well-defined ferromagnetic transitions with an increasing degree of hysteresis in the ferromagnetic state with decreasing Fe concentration. We also demonstrated that the cusp in the specific-heat anomaly associated with the ferromagnetic transition occurs very close to the magnetic ordering temperature. Furthermore, we showed evidence that the critical concentration for ferromagnetism is near 0.01 at. \% $\mathrm{Fe}$, or a factor of 10 less than previously assumed. As compared with the sharp magnetic transitions the specific-heat anomalies associated with the transitions are considerably broadened. We attribute this to fluctuations associated with the microscopic inhomogeneities in these random solid solutions.

\section{ACKNOWLEDGMENTS}

This work was supported by a grant from the Deutsche Forschungsgemeinschaft. One of us (H.C.) acknowledges the financial support of the Heraeus Stiftung.
${ }^{*}$ Permanent address: Department of Physics, University of Illinois at Chicago, P.O. Box 4348, Chicago, IL 60680.

${ }^{1}$ G. J. Nieuwenhuys, Adv. Phys. 24, 515 (1975); see also references therein.

${ }^{2}$ G. Griffith, F. A. Volkening, and H. Claus, J. Appl. Phys. 57, 3392 (1985).

${ }^{3}$ B. W. Veal and J. A. Rayne, Phys. Rev. B 5, 236 (1964).
${ }^{4}$ K. Albert, H. v. Löhneysen, W. Sander, and H. J. Schink, Cryogenics 22, 417 (1982).

${ }^{5}$ T. Auerswald, diplomarbeit, Universität Karlsruhe, 1990 (unpublished); see also, K. G. Vandervoort, J. Griffith, H. Claus, and G. W. Crabtree, Rev. Sci. Instrum. 62, 2271 (1991).

${ }^{6}$ S. Crane, D. W. Carnegie, and H. Claus, J. Appl. Phys. 53, 2179 (1982) 
${ }^{7}$ C. Büscher, diplomarbeit, Universität Karlsruhe, 1990 (unpublished).

${ }^{8}$ A. Pilipowicz and H. Claus, Phys. Rev. B 36, 773 (1987).

${ }^{9}$ A. Schröder, dissertation, Universität Karlsruhe, 1991 (unpublished).

${ }^{10}$ A. Pilipowicz, F. Volkening, G. Griffith, and H. Claus (unpublished); F. Volkening, Ph.D. thesis, University of Illinois at Chicago, 1988 (unpublished).

${ }^{11}$ R. P. Peters, Ch. Buchal, M. Kubota, R. M. Mueller, and F. Pobell, Phys. Rev. Lett. 53, 1108 (1984).

${ }^{12}$ D. I. Bradley, A. M. Guenault, V. Keith, C. J. Kennedy, S. G. Musset and G. R. Picket, in Proceeding of the Seventeenth International Conference on Low Temperature Physics, LT-17, edited by U. Eckern, A. Schmid, W. Weber, and H. Wühl (Elsevier, New York, 1984), p. 633.

${ }^{13}$ K. Kornik, H. Kunkel, and R. M. Roshko, J. Appl. Phys. 64,
5619 (1988), using our criterion to determine $T_{C}$ we obtain $T_{C}=0.32 \mathrm{~K}$ instead of $0.48 \mathrm{~K}$ as reported in this reference.

${ }^{14} \mathrm{At} 3.2$ at. $\% \mathrm{Fe}, T_{C}=101.5 \mathrm{~K}$, whereas the linear extrapolation of the straight line in Fig. 4 to 3.2 at. \% Fe would yield $T_{C}=133 \mathrm{~K}$.

${ }^{15}$ I. Y. Korenblit and E. F. Shender, Zh. Eksp. Teor. Fiz. 62, 1949 (1972) [Sov. Phys. JETP 35, 1017 (1972)].

${ }^{16}$ D. W. Carnegie, C. J. Tranchita, and H. Claus, J. Appl. Phys. 50, 7318 (1979).

${ }^{17}$ G. J. Nieuwenhuys, Phys. Lett. 43A, 301 (1973).

${ }^{18}$ R. A. Webb, G. W. Crabtree, and J. J. Vuillemin, Phys. Rev. Lett. 43, 796 (1979).

${ }^{19}$ B. H. Verbeek, G. J. Nieuwenhuys, J. A. Mydosh, C. van Dijk, and B. D. Rainford, Phys. Rev. B 22, 5426 (1980).

${ }^{20}$ J. C. Ododo, Solid State Commun. 25, 25 (1978).

${ }^{21}$ G. R. Stewart and B. L. Brandt, Phys. Rev. B 28, 2266 (1983). 